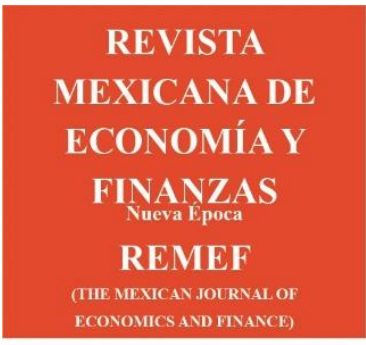

Revista Mexicana de Economía y Finanzas, Nueva Época

Volumen 16 Número 1, Enero - Marzo 2021, pp. 1-15, e581

DOI: https://doi.org/10.21919/remef.v16i1.581

(Recibido: 24/agosto/2020, aceptado: 27/noviembre/2020, publicado: 30/noviembre/2020)

\title{
Tendencias y perspectivas de la ciencia financiera: Un artículo de revisión
}

\author{
Robert Cox Merton ${ }^{1}$ - Massachusetts Institute of Technology, U.S.A. \\ Francisco Venegas-Martínez ${ }^{2}$ - Instituto Politécnico Nacional, México
}

Las contribuciones de Robert C. Merton cobran vigencia actual. Con respecto del diseño de los planes para el retiro, en un marco de sistemas deficientes de pensiones, las investigaciones de Merton (1969) y (1971) sobre reglas óptimas de consumo y portafolio durante y después de la vida laboral cobran vigencia contemporánea. Asimismo, Bodie y Merton (2002) proponen el uso de productos derivados, en el ámbito internacional, para diversificar los riesgos de los sistemas de pensiones justo en el momento en que muchos de éstos, en economías subdesarrolladas e industrializadas, están en la antesala de colapso; además de que varios de estos sistemas sólo garantizan una proporción exigua del salario. Por último, la teoría racional de valuación de opciones de Merton (1973) se retoma para crear oleoductos y plantas generadoras de energía sintéticas mediante el uso de contratos derivados. Este trabajo pretende realizar una revisión de las tendencias y perspectivas de la ciencia financiera y las finanzas matemáticas, en el marco de las contribuciones pioneras de Robert Cox Merton, resaltando áreas prioritarias que ofrecen oportunidades para realizar investigación con impactos social y global.

Clasificación JEL: D11, G12, G13, E43.

Palabras clave: ciencia financiera, decisiones de consumo y portafolio, productos derivados, estructuras de plazos de tasas de interés.

\section{Financial Science Trends and Perspectives: A Review Article}

Robert C. Merton's contributions are current. Regarding the design of retirement plans, in a framework of pension deficient systems, the investigations of Merton (1969) and (1971) on optimal consumption and portfolio rules during and after working life acquire contemporary validity. Likewise, Bodie and Merton (2002) propose the use of derivative products, at the international level, to diversify the risks of pension systems just at the moment when these systems of many underdeveloped and industrialized economies are on the verge of collapse; knowing that several of these systems only provide a meager proportion of the salary. Finally, Merton's theory of rational option pricing is retaken to create synthetic oil pipelines and power plants through the use of contingent claims. This paper aims to review the trends and perspectives in financial science and mathematical finance, within the framework of the pioneering contributions of Robert Cox Merton, highlighting priority areas that offer opportunities for research with social and global impacts.

JEL classification: D11, G12, G13, E43.

Keywords: financial science, consumer and portfolio decisions, derivative products, interest rate term structures.

1 Es profesor en el Sloan School of Management, Massachusetts Institute of Technology. Fue también profesor de la Universidad de Harvard, rmerton@mit.edu

2 Autor de correspondencia. Es profesor de la Escuela Superior de Economía, Instituto Politécnico Nacional, fvenegas1111@yahoo.com.mx

*Sin fuente de financiamiento para el desarrollo de la investigación. 


\section{Introducción}

Un aspecto relevante de las contribuciones de Robert Cox Merton es que todas ellas cobran vigencia actual. Por ejemplo, en el tema del diseño de planes para el retiro, las investigaciones de Merton (1969) y (1971) sobre reglas óptimas de consumo y portafolio durante y después de la vida laboral cobran vigencia, sobre todo a sabiendas de que los sistemas de pensiones actuales sólo garantizan una proporción exigua del salario (Merton, 2006). También cobra vigencia el uso de contratos derivados, en el ámbito internacional, para diversificar los riesgos en los sistemas de pensiones sin que se modifiquen los patrones y el comportamiento del empleo, la estructura industrial y el funcionamiento del sistema financiero (Bodie y Merton, 2002). Además, de Merton (1973) se retoma su teoría racional de valuación de opciones para crear oleoductos y plantas generadoras de energía sintéticas mediante el uso de productos derivados para hacer más eficiente el uso de los recursos energéticos $\mathrm{y}$, con ello, contar con un mundo más verde; siendo el nexo entre energía y medio ambiente uno de los más importantes en el ámbito global

Este trabajo pretende hacer una revisión de las tendencias de la ciencia financiera y las finanzas matemáticas con base en las contribuciones de R. C. Merton que sorprendentemente permanecen vigentes. Así como algunas otras contribuciones recientes en la administración de riesgos en el lavado de dinero con técnicas y herramientas de ciencia de datos y la evaluación del impacto de los derivados de tipo de cambio en ambientes de alta volatilidad (como la generada por el COVID-19) en los mercados bursátiles emergentes, así como la administración del riesgo de microcréditos en un ambiente de incertidumbre generada por la pandemia COVID-19 con herramientas y técnicas de ciencia de datos. ${ }^{3}$ Por último se destacan las áreas que ofrecen oportunidades para realizar investigación con impactos social y global en el marco de las contribuciones pioneras de Robert Cox Merton.

El presente trabajo está organizado de la siguiente forma: en la sección 2 se describe, brevemente, la evolución y vigencia de las aportaciones de R. C. Merton; en la sección 3 se discute sobre el impulso que las innovaciones financieras dieron a la ciencia financiera para que ésta entrara en un proceso maduración, resaltando la contribución de los productos derivados, y destacando cómo éstos, actualmente, se pueden utilizar para hacer más eficiente la política energética y también resaltando cómo se pueden utilizar para diversificar los riesgos en los sistemas pensionarios; en la sección 4 se presentan otras contribuciones sobre la gestión de diversos riesgos; en la sección 5 se establecen líneas de investigación futura con impactos social y global con base en las contribuciones de Merton; por último, en la sección 6 se dan las conclusiones.

\section{La evolución de las contribuciones de R. C. Merton}

Merton (1973) en su artículo pionero deduce un conjunto de condiciones necesarias que debe satisfacer la prima de una opción. Las condiciones están basadas en el supuesto de que los inversionistas prefieren más a menos (digamos, una teoría racional de precios). Merton se concentra también en activos subyacentes que pagan dividendos y cuando los términos del contrato de opción

${ }^{3}$ Martínez-Sánchez et al. (2020) y Bernal-Ponce et al. (2020), Rodríguez-García y Venegas-Martínez (2020). 
pueden modificarse explícitamente por un cambio en el precio de ejercicio o implícitamente por modificaciones en la política de inversión o estructura de capital de la empresa. Dado que las condiciones propuestas por Merton no son suficientes para determinar de manera única la prima de una opción, Merton hace supuestos adicionales para extender la fórmula de Black-Scholes (1973). En su trabajo, Merton obtiene fórmulas explícitas para la prima de opciones europeas de compra y venta, así como para warrants y opciones "down-and-out”. Merton (1973) examina además los efectos de los dividendos sobre el precio del colateral. También extiende la teoría de determinación de precios de los pasivos corporativos. Otras contribuciones no menos importantes de Merton en su artículo de 1973 son: el análisis de opciones americanas, la generalización de la fórmula de Samuelson para opciones perpetuas, la valuación de opciones con barreras y la introducción de tasas de interés estocásticas. Una diferencia esencial con el modelo clásico de Black y Scholes (1973) es que Merton no supone una tasa de interés constante y libre de riesgo de incumplimiento, sino los rendimientos de un bono cupón cero conducidos por un proceso de difusión, lo cual genera una estructura de plazos para la tasa de interés. ${ }^{4}$

Otros dos artículos pioneros de Robert C. Merton (1969) y (1971) tratan sobre el problema del consumidor racional que maximiza su utilidad total descontada cuando la dinámica de los rendimientos de los activos son conducidos por procesos de difusión. ${ }^{5}$ Sin duda, un tema de actualidad son los planes para el retiro en los que los agentes deben seguir reglas óptimas de consumo y portafolio durante la vida laboral, previendo que los sistemas de pensiones sólo garantizan una proporción exigua del salario (Merton, 2006). En este sentido, Merton hace las siguientes recomendaciones para planear el retiro: 1) considerar los riesgos asociados a los rendimientos de lo que quieres recibir; 2 ) determinar una estrategia de inversión que asuma el riesgo a que te quieras exponer; 3 ) determinar las reglas óptimas sobre los activos antes y después de pensionarte; 4) tomar en cuenta los cambios del mercado; 5) plantear una estrategia de inversión de bajo costo (comisiones). ${ }^{6}$

Por otro lado, gran parte de las extensiones de los trabajos de Merton (1969) y (1971) apuntan hacia el modelado del comportamiento estocástico de los precios de los activos. Por ejemplo cuando se presentan con cambios de régimen, en tendencia y/o volatilidad, definidos por una cadena de Markov. En este sentido, Bäuerle y Rieder (2004) determinan las decisiones óptimas de portafolio óptimo cuando el precio de las acciones depende de una cadena de Markov finita y homogénea en el tiempo y Sotomayor y Cadenillas (2009) encuentran soluciones explícitas para las decisiones óptimas de inversión y consumo con una función de utilidad HARA (Hyperbolic Absolute Risk Aversion) cuando los precios de los activos son conducidos por movimientos brownianos estándar combinados con un cambio de régimen markoviano. Y, por último, Fei (2013) proporciona las

\footnotetext{
${ }^{4}$ Es importante destacar aquí que Black y Scholes (1973), bajo un conjunto de supuestos, determinan la prima de una opción europea de compra siguiendo caminos totalmente diferentes al de Merton (1973): la ecuación de difusión de calor y el modelo Capital Asset Picing Theory (CAPM) en una versión en tiempo continuo. Otra contribución importante que hay que tener en cuenta sobre crecimiento económico estocástico se encuentra en Merton (1975).

5 La selección óptima de portafolio se ha estudiado ampliamente en Biagini y Øksendal (2003), Czichowsky y Schachermayer (2015), Hu y Øksendal. (2003), Hu et al. (2003), Jumarie (2005), Karatzas et al. (1987), He y Pearson (1991), Karatzas et al. (1991), Cvitani' y Karatzas (1996), Cvitani' y Wang (2001), Venegas-Martínez (2001), (2005) y (2009), Venegas-Martínez y González-Aréchiga (2000), y Zariphopoulou (2001), (1999) y (1992).

${ }^{6}$ En la liga https://scholar.google.es/scholar?start=10\&q=robert+c+merton\&hl=es\&as_sdt=0,5 aparecen todas las publicaciones de Robert C. Merton,
} 
decisiones óptimas de consumo y de portafolio cuando la tasa de inflación es conducida por un proceso de cambio de régimen Markoviano.

Otros trabajos, no menos importantes, que han estudiado el problema de la determinación de decisiones óptimas de consumo y portafolio con modelos de cambio de régimen markoviano son, por ejemplo: Stockbridge (2002) que proporciona una formulación de programación matemática del problema de optimización de portafolio; Zhang y Yin (2004) que ofrecen estrategias casi-óptimas en un mercado financiero; Sass y Haussmann (2004) quien resuelven numéricamente el problema de maximización de utilidad esperada del inversionista de la riqueza final en un horizonte de finito tiempo; y Vallejo-Jiménez et al. (2015) y Soriano-Morales et al. (2015) que combinan cambio de régimen markoviano con múltiples saltos. ${ }^{7}$

Vale la pena hacer una pequeña lista, que presentamos en el Cuadro 1. En esta lista se destaca una parte del desarrollo teórico que el trabajo pionero de Merton (1969) y (1971) ha impulsado en la literatura especializada sobre el consumidor-inversionista racional en tiempo continuo con rendimientos de activos conducidos por difusiones. Por supuesto, esta lista no pretende, en lo absoluto, ser exhaustiva.

Tabla 1. Impulso del trabajo pionero de Merton (1969) y (1971)

\begin{tabular}{|l|l|}
\hline Proceso de diffusion & Merton (1969) y (1971) \\
\hline Proceso de difusión con saltos & $\begin{array}{l}\text { Czichowsky y Schachermayer (2015), Jin y } \\
\text { Zhang (2012), Aït-Sahalia et al. (2009), } \\
\text { Venegas-Martínez (2000) y (2001), } \\
\text { Jeanblanc-Picqué y Pontier (1990). }\end{array}$ \\
\hline $\begin{array}{l}\text { Proceso de difusión modulado por } \\
\text { una cadena finita de Markov } \\
\text { homogénea en el tiempo }\end{array}$ & $\begin{array}{l}\text { Soriano-Morales et al. (2015), Fei (2014), } \\
\text { Zhou y Yin (2014), Wu y Li (2011), Elliott et al. } \\
\text { (2010), Sotomayor y Cadenillas (2009), } \\
\text { CCakmak y Özekici (2006), Rieder y Bäuerle } \\
\text { (2005), y Bäuerle y Rieder (2004), Sass } \\
\text { (2004), Sass y Haussmann (2004), } \\
\text { Stockbridge (2002), y Elliot (2002). }\end{array}$ \\
\hline $\begin{array}{l}\text { Proceso de difusión modulados por } \\
\text { una cadena finita de Markov no } \\
\text { homogénea en el tiempo }\end{array}$ & $\begin{array}{l}\text { Vallejo-Jiménez et al. (2015), y Rudiger y } \\
\text { Backhau (2008). }\end{array}$ \\
\hline $\begin{array}{l}\text { Movimiento fraccional browniano } \\
\text { modulado por una cadena de Markov } \\
\text { finita homogénea en el tiempo }\end{array}$ & \begin{tabular}{l} 
Fei y Shu-Juan (2012). \\
\hline
\end{tabular} \\
\hline
\end{tabular}

\footnotetext{
${ }^{7}$ Hasta donde sabemos, los modelos de cambio de régimen fueron propuestos inicialmente por Hamilton (1990. Además, los primeros en tratar los precios de los activos conducidos por procesos mixtos de difusión con saltos fueron: Cox y Ross (1976), Ball y Torous (1985) y Page y Sanders (1986). Se puede encontrar un trabajo más reciente sobre el proceso de difusión por salto en Aït-Sahalia et al. (2009) y Lui et al. (2005). El movimiento browniano fraccional es una extensión natural del movimiento browniano (Mandelbrot, 1968) y sus propiedades estadísticas se utilizan ampliamente en modelos financieros; ver, por ejemplo, Bender et al. (2011) y Hu y Øksendal (2003).
} 


\begin{tabular}{|l|l|}
\hline $\begin{array}{l}\text { Proceso de difusión con saltos } \\
\text { modulado por una cadena de Markov }\end{array}$ & Yu (2014), y Elghanjaoui y Karlsen (2012). \\
\hline $\begin{array}{l}\text { Movimiento fraccional browniano } \\
\text { combinado con múltiples procesos - } \\
\text { saltos modulado por una cadena de } \\
\text { Markov no homogénea en el tiempo }\end{array}$ & Vallejo-Jiménez y Venegas-Martínez (2017). \\
\hline
\end{tabular}

Fuente: Elaboración de los autores

\section{Sistemas financieros, innovaciones y ciencia financiera}

Para el mejor funcionamiento de los sistemas financieros es esencial la innovación financiera, la cual incrementa la eficiencia de estos sistemas a través de nuevos instrumentos financieros y del uso de modelos, técnicas, herramientas y metodologías novedosas, todo junto con el avance de las tecnologías de información.

Las nuevas propuestas de diversos modelos en las décadas de los cincuentas y los sesentas contribuyeron a que las finanzas se empezaran a convertir en una en ciencia formal. Posteriormente, la ciencia financiera y la práctica financiera se conectaron inexorablemente en las décadas de los setentas y los ochentas con la creación de diversos instrumentos, dentro de los cuales destacan los productos derivados. Algunos eventos innovadores que ayudaron a que las finanzas se convirtieran en ciencia entre 1950 y 1960 son los siguientes:

- 1952, la diversificación mediante la teoría de la portafolios con el enfoque media-varianza de Harry Markowitz.

- 1953, el papel de los activos financieros en la asignación óptima del riesgo de Kenneth Joseph Arrrow.

- 1958, la preferencia de liquidez como comportamiento frente al riesgo de James Tobin.

- 1958, la estructura óptima de capital en finanzas corporativas y la política de dividendos de Franco Modigliani and Merton H. Miller.

- 1960, la primera base de datos integral de rendimiento de acciones individuales del Centro de Investigación de Precios de Activos de Chicago.

- 1963 y 1965, la Hipótesis del Mercado Eficiente de Eugene Fama y Paul Samuelson.

- 1964, 1965 y 1966, las diferencias en los rendimientos esperados basadas en el riesgo de William Forsyth Sharpe - John Lintner - Jan Mossin - Jack L. Treinor, y el modelo de valuación de activos de capital (Capital Asset Pricing Model, CAPM).

- 1968 y 1969, la prueba del desempeño de varios inversionistas institucionales utilizando el CAPM - Michael C. Jensen y Richard Roll.

Por otro lado, las crisis pueden inducir la generación de innovaciones financieras que conduzcan a mejoras permanentes en el sistema financiero, tal y como sucedió en las décadas de los setentas y los ochentas. Una de las principales crisis financieras y económicas que se cuentan en la historia se dio en la década de los setentas en EE.UU con las siguientes características: 
- Explosión multidimensional de volatilidad en las economías occidentales reflejada en los sistemas financieros.

- Caída del sistema monetario de Bretton Woods.

- $\quad$ Primera crisis del petróleo en 1973-1974 y una segunda en 1979.

- Inflación de dos dígitos en EEUU, la más alta desde la Guerra Civil.

- Tasas de interés de dos dígitos, las más altas desde la Guerra Civil.

- Falta de disponibilidad de fondos hipotecarios: la regulación Q-5\% y el límite de pago de intereses en cuentas de depósito.

- La "estanflación” como una enfermedad económica, desconocida y aún sin solución.

- Caída del mercado de valores en un 50\% en términos reales en 1973-1974.

- La recesión de 1973-1975 y sus estragos hasta la década de los ochentas.

Esta explosión de riesgo en EEUU en la década de los setentas condujo hacia una explosión de innovaciones financieras, adoptada posteriormente en todo el mundo. Durante este período de tiempo, la práctica y la teoría financieras se vinculan como se muestra con los siguientes hechos:

- Mercado sobre mostrador de opciones.

- Futuros financieros para divisas, tasas de interés y acciones.

- NASDAQ, primer mercado de valores automatizado y electrónico.

- Fondos del mercado de dinero, bonos de alto rendimiento de tasa flotante.

- Fondos indexados, Stage Coach Fund 1970 y Vanguard 1975.

- Diversificación internacional de acciones de TIAA-CREF 1972.

- Sistema moderno de pensiones ERISA 1974, financiado por los empleadores en EEUU.

- El 1 de mayo de 1975 se permite el cobro de comisiones variables sobre la compra y venta de acciones.

- Titularización de deuda y creación de un mercado hipotecario nacional.

- Eliminación de regulaciones destructivas: límites máximos a las tasas de depósito.

- Establecimiento de las bases para la globalización de los mercados de capitales: mercados de derivados adoptados en casi todo el mundo y, a través de ellos, diversificación global.

- Madures de la ciencia financiera: Tanto los modelos cuantitativos existentes como los novedosos fueron utilizados ampliamente en los sistemas financieros.

- Aparecen los primeros departamentos (o facultades) de Finanzas y pregrados y posgrados en Finanzas

Una pregunta importante con respecto a estas innovaciones es ¿Cómo los contratos de derivados intangibles pueden resolver desafíos tangibles para el crecimiento económico y la estabilización? Los contratos de derivados pueden redistribuir los riesgos en forma no invasiva y reversible. A continuación mencionamos algunas características especiales de la relación entre los mercados de derivados y la innovación financiera y, por supuesto, destacamos la maduración de la ciencia financiera que se dio con los productos derivados. 
- Los derivados son "adaptadores" eficientes entre sistemas financieros heterogéneos que mejoran la integración y diversificación financiera global.

- Los derivados proporcionan una implementación eficiente de los tres métodos de administración del riesgo: diversificación, cobertura y aseguramiento.

- El desarrollo de los mercados de derivados para acciones, tasas de interés, divisas, materias primas y bienes básicos (commodities) promueven la estabilidad financiera a través de múltiples canales para la transferencia de riesgos y la extracción de información de los precios.

- Los derivados pueden mejorar la eficiencia de las operaciones de estabilización y de mercado abierto: negociación eficiente y emisión de valores de "política de mercado abierto".

- Regulación informada para aprovechar los beneficios de la innovación financiera mientras se administran riesgos.

\subsection{Algunos casos exitosos de mayor eficiencia y un mundo más limpio}

Durante décadas se han sustituido contratos por activos físicos para crear una mayor eficiencia y un mundo más limpio, como fueron los casos del Gasoducto de Leipzig, en Alemania, y el Valle de Tennessee, en EEUU, la mayor empresa de energía pública EEUU, tal y como se muestra a continuación. Estas experiencias tienen que ser retomadas en la actualidad para hacer más eficientes los recursos energéticos y contar con un mundo más verde.

\subsubsection{Gasoducto de Leipzig}

Consideremos el gasoducto de Leipzig en la década de los noventas en donde se utilizaron contratos derivados que jugaron el papel de un oleoducto sintético para crear una fuente de energía más eficiente en un mundo más limpio. La reunificación alemana en 1990 generó un rápido desarrollo económico y una mayor demanda de energía. Para satisfacer esta demanda se requirió una mayor oferta de gas natural. Leipzig tenía dos opciones que se muestran en la Figura 1 y el Cuadro 1.

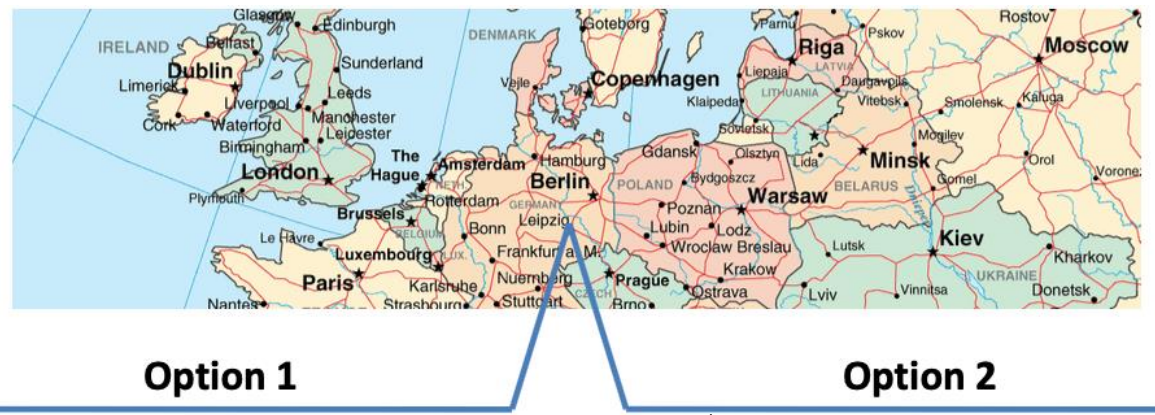

Gastar \$ 50 millones por un gasoducto hacia la red de gas europea y comprar gas del Reino Unido, Noruega y Holanda a precios de mercado indexados al precio del combustible para calefacción en dólares americanos (USD) en el punto de entrega del alto Rin.

Gastar \$ 300 millones en un nuevo gasoducto para conectarse a la red de gas rusa y celebrar un contrato a precio fijo por 15 años en marcos alemanes.

Figura 1. Las opciones del Gasoducto de Leipzig 
Cuadro 1. Síntesis contractual de activos: Gasoducto de Leipzig.

\begin{tabular}{|l|l|l|}
\hline & Opción 1 & Opción 2 \\
\hline Inversión de capital & $\$ 50 \mathrm{M}$ & $\$ 300 \mathrm{M}$ \\
\hline Ventajas & $\begin{array}{l}\text { Riesgo político reducido al } \\
\text { evitar la dependencia de los } \\
\text { rusos. } \\
\text { Menor inversión de capital. }\end{array}$ & $\begin{array}{l}\text { Precios estables de energía } \\
\text { potencialmente útiles para la } \\
\text { población acostumbrada a los } \\
\text { controles de precios. }\end{array}$ \\
\hline Desventajas & Volatilidad del precio del gas. & Alta inversión de capital. \\
\hline
\end{tabular}

La opción 1 de cobertura tenía dos problemas importantes por resolver:

1. Limitaciones de los instrumentos de cobertura disponibles:

- Opción de compra de petróleo crudo de hasta por 5 años en USD.

- Swaps de crudo/combustible de calefacción hasta por 2 años.

- Opciones de divisas hasta por 5 años.

- $\quad$ Swaps de divisas hasta por 10 años.

2. Sofisticación limitada de la administración de la ciudad.
Solución eficiente y ecológica

Un banco emitió una opción a 15 años a los precios europeos del gas a un precio de ejercicio igual al contrato de precio fijo ruso a cambio de una prima de 125 millones. La opción es efectivamente un oleoducto sintético".

El precio fue la mitad del costo incremental de un oleoducto físico para Rusia y compensa al banco por los desajustes de cobertura y la necesidad de ajustar dinámicamente las coberturas durante 15 años.

\subsubsection{La Autoridad del Valle de Tennessee}

Otro caso que hay que resaltar es el de la Tennessee Valley Authority (TVA) que utilizó contratos de opciones de compra para comprar energía como alternativa a la construcción de nuevas plantas generadoras. Es decir, los contratos de opción jugaron el papel de plantas generadoras. De esta manera, TVA no construyó dos plantas de energía nuclear, equivalentes a 35 convencionales.

- En 1994, Tennessee Valley Authority (TVA), la mayor empresa de energía pública de EEUU llevó a cabo un análisis estratégico a largo plazo de las demandas de energía de sus clientes y desarrolló canales de suministro robustos que no se vieron afectados por choques externos por la flexibilidad ofrecida.

- TVA adoptó una propuesta innovadora para satisfacer las necesidades de capacidad incremental mediante el uso de contratos derivados para comprar energía como alternativa a la construcción de nuevas plantas generadoras.

- La propuesta de TVA consistía en la creación de contratos de opciones de compra a largo plazo sobre energía con contrapartes que pudieran entregar la energía a la red.

- Como consecuencia de la implementación del acuerdo, TVA no construyó dos plantas de energía nuclear, equivalentes a 35 convencionales. 
- En 2016, TVA fue responsable del 3.5\% de toda la generación de energía eléctrica en EEUU.

\subsection{Swaps de pensiones en el ámbito internacional}

Las lecciones de la experiencia internacional han mostrado diversos beneficios de los swaps para diversificar el riesgo de los sistemas de pensiones. Dichos beneficios pueden enumerarse de la siguiente manera:

- Menor costo de capital a través de una mayor adquisición de riesgo global de los riesgos de un país.

- Contrapartes naturales siempre disponibles: si un país tiene "demasiada" exposición al riesgo para sí mismo por diversificación, el resto del mundo tiene "muy poca" exposición al riesgo en ese país.

- Implementación de bajo costo. Las transacciones se realizan directamente entre fondos soberanos, fondos de pensiones del gobierno, reservas y bancos centrales, sin necesidad de incurrir en costos de intermediación ni riesgo crediticio.

- Reducción del riesgo moral de expropiación, repudio, impuestos o contabilidad.

- Otorgan garantía de crédito y/o colateral de dos vías con valor de mercado.

En los últimos años, los productos derivados, particularmente los contratos swaps se han convertido en dispositivos financieros que conectan diversos sistemas financieros nacionales con la red financiera mundial. En la actualidad, los sistemas financieros de todo el mundo utilizan ampliamente swaps (de tasas de interés y tipo de cambio) para administrar sus riesgos de mercado y disminuir sus costos de transacción (comisiones). Sin embargo, la gran mayoría de los fondos de pensiones que han crecido rápidamente durante los últimos 20 años, escasamente utilizan swaps cuando éstos podrían utilizar swaps para diversificar sus riesgos en un ámbito internacional. Las ventajas relativas que ofrecen lo swaps en la cobertura de fondos de pensiones son:

- Los residentes realizan la gobernanza industrial, la negociación de acciones en el mercado local y la distribución de intermediación local de las exposiciones a los rendimientos de activos globales.

- El uso de estos derivados es robusto con respecto al diseño del sistema financiero local: funciona con políticas de estabilización financiera (incluidos los controles de capital).

- La política no es invasiva: no requiere cambios en los patrones y comportamiento del empleo, cambios en la estructura industrial o cambios en el diseño del sistema financiero.

- La póliza es reversible simplemente entrando en un swaption de compensación.

\section{Otras contribuciones recientes sobre gestión del riesgo}

El lavado de dinero, no sólo de actividades criminales, sino también del desvío de recursos públicos, es uno los problemas que se encuentran presentes en todo el mundo. Al respecto, el trabajo de Martínez-Sánchez, Cruz-García y Venegas-Martínez (2020) tiene como objetivo desarrollar un modelo de árbol de regresión útil para cuantificar el riesgo asociado al lavado de dinero (LD) 
considerando el perfil del cliente y sus productos contratados (riesgo inherente del cliente). En el riesgo de LD hay varias instituciones y entidades expuestas, pero principalmente las financieras por la naturaleza de su actividad, de manera que están legalmente obligadas a contar con una metodología adecuada para analizar y evaluar dicho riesgo. La investigación desarrollada utiliza la técnica de árboles de regresión (una técnica de ciencia de datos) para identificar, medir y cuantificar el riesgo del LD inherente del cliente. Después de clasificar a los clientes en alto o bajo riesgo según un umbral de probabilidad de 0.5. El trabajo encuentra que: los clientes con 56 meses o más de antigüedad son más riesgosos que aquellos con menos antigüedad; las variables "producto contratado" y "antigüedad del cliente" son estadísticamente significativas; la entidad legal y la actividad económica no son estadísticamente significativas para clasificar a los clientes; los productos comerciales y los productos individuales son los más riesgosos; y el porcentaje de efectividad, sugerido por la técnica del árbol de decisiones es de alrededor del 89.5\%. En la práctica diaria de la gestión de riesgos de LD, los dos problemas principales son: 1) el conocimiento del cliente, y 2) la detección de sus elementos de riesgo inherentes

Otro trabajo de Bernal-Ponce, Castillo-Ramírez y Venegas-Martínez (2020) investiga el efecto de los derivados sobre la relación entre los tipos de cambio y los mercados de valores. El trabajo utiliza un modelo teórico de un inversionista racional y realiza un análisis empírico para las bolsas de valores de México y Brasil en 2007-2019. Los resultados del modelo teórico se aplican a la gestión de carteras internacionales, proponiendo una estrategia para mitigar la exposición cambiaria con derivados. Este estudio contribuye a la literatura al explicar cómo el índice de cobertura de varianza mínima juega un papel esencial en el nexo de divisas y mercados bursátiles.

Por último, el otorgamiento de microcréditos de forma eficiente y transparente a través de plataformas digitales a individuos que desarrollan actividades económicas y que buscan mantener su empleo y el de sus trabajadores y que no tienen acceso al sistema financiero convencional es, sin duda, un problema urgente por resolver en la crisis sanitaria mundial. El trabajo de Rodríguez-García y Venegas-Martínez (2020) desarrolla varios modelos y estrategias de riesgo de crédito que permiten promover la inclusión crediticia en México de manera justa y sostenible en un ambiente de incertidumbre generada por los estragos presentes y esperados por la pandemia COVID-19. Para ello se utiliza el enfoque de ciencia de datos de machine learning, particularmente, árboles de regresión, bosques aleatorios, Boosting, K-Nearest Neigbor (KNN) y redes neuronales. ${ }^{8}$

\section{Propuestas de investigación futura con impactos social $y$ global}

Con base en el desarrollo de las secciones anteriores se presentan a continuación algunas de las áreas que ofrecen oportunidades para realizar investigación con impactos social y global en el marco de las contribuciones pioneras de Merton; la lista no pretende ser exhaustiva,

\footnotetext{
${ }^{8}$ En la liga

https://econpapers.repec.org/scripts/search.pf?ft=\&adv=true\&wp=on\&art=on\&bkchp=on\&soft=on\&pl=\&auth=on\&sort= rank\&lgc $=$ AND\&aus=venegas $\& a r=0 n \& k w=\& j e l=\& n e p=\& n i=\&$ nit=epdate

aparecen alrededor de 20 libros de F. Venegas-Martínez relacionados con temas de Finanzas.
} 
- Derivados de energía para generar oleoductos, gasoductos y plantas generadoras de energía "sintéticas" con respeto al medio ambiente.

- Reglas óptimas de consumo y portafolio con costos de transacción.

- Reglas óptimas de consumo y portafolio con procesos de Lévy combinados con procesos de Cox modulados por cadenas de Markov.

- Reglas óptimas de consumo y portafolios durante y después de la vida laboral de un individuo.

- Diversificación de riesgos en sistemas pensionarios con swaps en el ámbito internacional.

- Administración del riesgo con múltiples factores de microcréditos en el entorno de la pandemia COVID-19.

- Desarrollo de modelos macroeconómicos estocásticos con sector salud y su vulnerabilidad a eventos extremos (catástrofes, pandemias -COVID-19-, etc.)

- Efectos de la incertidumbre en la política económica en los mercados financieros.

- Control del riesgo de lavado de dinero con múltiples factores.

- Nexo entre los sectores financiero y energético para contar con un mundo más verde.

- Comportamiento de agentes financieros (finanzas conductuales) e inteligencia artificial.

- Ciencia de datos aplicada a banca y negocios

- Análisis de los efectos de la pandemia COVID-19 en los mercados financieros con herramientas de ciencia de datos.

\section{Conclusiones}

En el transcurso de este trabajo se realizó una revisión de las tendencias y perspectivas de la ciencia financiera y las finanzas matemáticas con base en las contribuciones de R. C. Merton, las cuales permanecen vigentes, así como algunas otras contribuciones recientes en administración de riesgos. Se hizo también un seguimiento detallado de la evolución de la ciencia financiera y de la importancia que tiene la innovación financiera, particularmente los productos derivados, lo cual ha incrementado la eficiencia de los sistemas financieros junto con el avance de las tecnologías de información. Por último, la investigación presenta un diagnóstico sobre las áreas que ofrecen oportunidades para realizar investigación tanto con impacto social como global.

\section{Referencias}

[1] Aït-Sahalia, Y., J. Cacho-Diaz, and T. Hurd (2009). Portfolio Choice with Jumps: A Closed-Form Solution. Annals of Applied Probability, 19(2), 556-584.

[2] Arrow K. J. (1953). Le rôle des valeurs boursières pour la répartition la meilleure des risques. Econometrie 11:41-47. Trad. 1964. The role of securities in the optimal allocation of risk-bearing. Review of Economic Studies. 31(2), 91-96.

[3] Ball, C. A., and W. N. Torous (1985). On Jumps in Common Stock Prices and Their Impact on Call Option Pricing. Journal of Finance, 40(1), 155-173.

[4] Bäuerle, N. and U. Rieder (2004). Portfolio Optimization with Markov-Modulated Stock Prices and Interest Rates. IEEE Transactions on Automatic Control, 49(3), 442-447.

[5] Bender, C., T. Sottinen and E. Valkeila (2011). Fractional Processes as Models in Stochastic Finance. Advanced Mathematical Methods for Finance. Springer, Berlin, Heidelberg. 
[6] Bernal-Ponce, L. A., C. E. Castillo-Ramírez and F. Venegas-Martínez (2020). Impact of Exchange Rate Derivatives on Stocks in Emerging Market. Journal of Business Economics and Management, 21(2), 610-626.

[7] Black, F. and R. Litterman (1992). Global Portfolio Optimization. Financial Analysts Journal, 48(5), 2843.

[8] Black, F., and R. Litterman (1990), Asset Allocation: Combining Investor Views with Market Equilibrium, Goldman Sachs Fixed Income Research.

[9] Bodie, Z. and R. C. Merton (2002). International Pension Swaps. Journal of Pension Economics and Finance, March 2002, 77-83.

[10] Çakmak, S., and S. Özekici (2006). Portfolio Optimization in Stochastic Markets. Mathematical Methods of Operations Research, 63(1), 151-168.

[11] Cao, M. (2001). Systematic Jump Risks in a Small Open Economy: Simultaneous Equilibrium Valuation of Options on the Market Portfolio and the Exchange Rate. Journal of International Money and Finance, 20(2), 191-218.

[12] Chandrasekhar Reddy Gukhal, C. R. (2004). The Compound Option Approach to American Options on Jump-Diffusions. Journal of Economic Dynamics and Control, 28(10), 2055-2074.

[13] Czichowsky, C., and W. Schachermayer (2015). Portfolio Optimisation Beyond Semimartingales: Shadow Prices and Fractional Brownian Motion.

[14] Elliott, R. J., T. K. Siuc, A. Badescud (2010). On Mean-Variance Portfolio Selection under a Hidden Markovian Regime-Switching Model. Economic Modelling, 27(3), 678-686.

[15] Elliott, J. R.., and T. K. Siu (2010). On Risk Minimizing Portfolios under a Markovian Regime-Switching Black-Scholes Economy. Annals of Operations Research, 176(1), 271-291.

[16] Elghanjaoui, S., and K. H. Karlsen (2012). A Markov Chain Approximation Scheme for a Singular Investment-Consumption Problem with Levy Driven Stock Prices. Working paper.

[17] Elliot, R. (2002). Portfolio Optimization, Hidden Markov Models, and Technical Analysis of P\&F-Charts. International Journal of Theoretical and Applied Finance, 5(4), 385-399.

[18] Fama, E. (1965). The Behaviour of Stock Market Prices. Journal of Business, 64(1), 34-105.

[19] Fei W., and L. Shu-Juan (2012). Study on Optimal Consumption and Portfolio with Inflation under Knightian Uncertainty. Chinese Journal of Engineering Mathematics, 2012-06.

[20] Fei, W. Y. (2013). Optimal Consumption and Portfolio under Inflation and Markovian Switching. Stochastics, 85(2), 272-285.

[21] Fei, W. Y. (2014). Optimal Control of Uncertain Stochastic Systems with Markovian Switching and Its Applications to Portfolio Decisions. Cybernetics and Systems, 45(1), 69-88.

[22] González-Aréchiga, B., J. Díaz-Tinoco y F. Venegas-Martínez (2001). Riesgo cambiario, brecha de madurez y cobertura con futuros: análisis local y de valor en riesgo. Economía Mexicana Nueva Época, 10(2), 259-290.

[23] Hamilton, J. D. (1990). Analysis of Time Series Subject to Changes in Regime. Journal of Econometrics, 45 (1-2), 39-70.

[24] Harrison, J. M. and D. M. Kreps (1979). Martingales and Arbitrage in Multiperiod Securities Markets. Journal of Economic Theory, 20(3), 381-408.

[25] $\mathrm{Hu}, \mathrm{Y} .$, and B. Øksendal. (2003). Fractional White Noise Calculus and Applications to Finance. Infinite Dimensional Analysis, Quantum Probability and Related Topics, 6(1), 1-32.

[26] Hu, Y., B. Øksendal, and A. Sulem (2003). Optimal Consumption and Portfolio in Black-Scholes Market Driven by Fractional Brownian Motion. Infinite Dimensional Analysis, Quantum Probability and Related Topics, 6(4), 519-536. 
[27] Jeanblanc-Picqué, M. and M. Pontier (1990). Optimal Portfolio for a Small Investor in a Market Model with Discontinuous Prices. Applied Mathematics and Optimization, 22(1), 287-310.

[28] Jensen, M. C. (1968). The Performance of Mutual Funds in the Period 1945-1964. Journal of Finance, 23(2), 389-416.

[29] Jensen, M. C. (1969). Risk, the Pricing of Capital Assets, and the Evaluation of Investment Portfolios. Journal of Business, 42(2), 167-247.

[30] Jin, X, and A. X. Zhang (2012). Decomposition of Optimal Portfolio Weight in a Jump-Diffusion Model and Its Applications. Review of Financial Studies, 25(9), 2877-2919.

[31] Jumarie, G. (2005). Merton's Model of Optimal Portfolio in a Black-Scholes Market Driven by a Fractional Brownian Motion with Short-Range Dependence. Insurance: Mathematics and Economics, 37(3), 585-598.

[32] Lintner, J. (1965). The Valuation of Risk Assets and the Selection of Risky Investments in Stock Portfolios and Capital Budgets, Review of Economics and Statistics, 47(1), 13-37.

[33] Lui, J., J. Pan, and T. Wang (2005). An Equilibrium Model of Rare-Event Premia and Its Implication for Option Smirks. Review of Financial Studies, 18(1), 131-164.

[34] Mandelbrot B. B. and J. W. Van Ness (1968). Fractional Brownian Motions, Fractional Noises and Applications. SIAM Review, 10(4), 422-437.

[35] Martínez-Sánchez, J. F., S. Cruz-García, and F. Venegas-Martínez (2020). Money Laundering Control in Mexico: A Risk Management Approach through Regression Trees (Data Mining). Journal of Money Laundering Control. 23(2), 427-439.

[36] Markowitz, H. (1952), Portfolio Selection. Journal of Finance, 7(1), 77-91.

[37] Merton, R. C. (1975). An Asymptotic Theory of Growth under Uncertainty. Review of Economic Studies, 42(3), 375-393.

[38] Merton, R. C. (2006). Allocating Shareholder Capital to Pension Plans. Journal of Applied Corporate Finance, 18(1), 15-24.

[39] Merton, R. C. (1973). Theory of Rational Option Pricing. The Bell Journal of Economics and Management Science, 4(1), 141-183.

[40] Merton, R. C. (1971). Optimum Consumption and Portfolio Rules in a Continuous-Time Model. Journal of Economic Theory, 3(4), 373-413.

[41] Merton, R. C. (1969). Lifetime Portfolio Selection under Uncertainty: The Continuous-Time Case. Review of Economics and Statistics, 51(3). 248-257.

[42] Merton, R. C. (1999). Country Risk. Commentary, Risk Magazine.

[43] Merton, R. C. (2002). Swapping Countries, Insights, ICBI 2002 Conference Highlights, PricewaterhouseCoopers.

[44] Merton, R. C (2004). Foreword: On Financial Innovation and Economic Growth. Harvard China Review, (Spring 2004), 2-3.

[45] Modigliani, F. and M. H. Miller (1958). The Cost of Capital, Corporation Finance and the Theory of Investment. American Economic Review, 48(3), 261-297.

[46] Mossin, J. (1966). Equilibrium in a Capital Asset Market. Econometrica, 34(4), 768-783.

[47] Page, F. H., and A. B. Sanders (1986). A General Derivation of the Jump Process Option Pricing Formula. Journal of Financial and Quantitative Analysis, 21(4), 437-446.

[48] Rieder, U. and N. Bäuerle (2005). Portfolio Optimization with Unobservable Markov-Modulated Drift Process. Journal of Applied Probability, 42(2), 362-378.

[49] Rodríguez-García, J. H. y F. Venegas-Martínez (2020). Reducción de la brecha del crédito en México en un ambiente de incertidumbre generada por la pandemia COVID-19: Un enfoque de ciencia de datos, Instituto Politécnico Nacional. México. Documento de trabajo. 
[50] Roll, R. (1969). Bias in Fitting the Sharpe Model to Time Series Data. Journal of Financial and Quantitative Analysis, 4, 271-89.

[51] Rudiger, F. and J. Backhau (2008). Pricing and Hedging of Portfolio Credit Derivatives with Interacting Default Intensities. Department of Mathematics, University of Leipzig, Working paper.

[52] Samuelson, P. (1965). Proof that Properly Anticipated Prices Fluctuate Randomly. Industrial Management Review, 6(2), 41-49.

[53] Sass, J. (2004). Optimizing the Terminal Wealth under Partial Information: The Drift Process as a Continuous Time Markov Chain. Finance and Stochastics 8(4), 553-577.

[54] Sass, J., and U. G. Haussmann (2004). Optimizing the Terminal Wealth under Partial Information: The Drift Process as a Continuous Time Markov Chain. Finance and Stochastics, 8(4), 553-577.

[55] Sharpe, W. (1964). Capital Asset Prices: A Theory of Market Equilibrium under Conditions of Risk, Journal of Finance, 19 (3), 425-442.

[56] Sharpe, W. F. (1963). A Simplified Model for Portfolio Analysis. Management Science, 9(2), 277-293.

[57] Sharpe, W. F. (1964). Capital Asset Prices: A Theory of Market Equilibrium under Conditions of Risk. Journal of Finance, 19(3), 425-442.

[58] Sharpe, W. F. (1966). Mutual Fund Performance. Journal of Business, 39(3), 119-138.

[59] Soriano-Morales, Y. V., F. Venegas-Martínez, y B. Vallejo-Jiménez (2015). Determination of the Equilibrium Expansion Rate of Money when Money Supply is Driven by a Time Homogeneous Markov Modulated Jump Diffusion Process. Economics Bulletin, 35(4), 2074-2084.

[60] Sotomayor, L. R. and A. Cadenillas (2009). Explicit Solutions of Consumption-Investment Problems in Financial Markets with Regime Switching. Mathematical Finance, 19(2), 251-279.

[61] Stockbridge, R. (2002). Portfolio Optimization in Markets Having Stochastic Rates. Lecture Notes in Control and Information Sciences: Stochastic Theory and Control, Vol. 280. Springer Verlag, Hidelberg.

[62] Téllez-León, I. E., F. Venegas-Martínez y A. Rodríguez-Nava (2011). Inflation Volatility and Growth in a Stochastic Small Open Economy: A Mixed Jump-Diffusion Approach. Economía, Teoría y Práctica, 35, 131-156.

[63] Tobin, J. (1958). Liquidity Preference as Behavior towards Risk. Review of Economic Studies, 25(2), 65-86.

[64] Treynor, J. L. (1965). How to Rate Management of Investment Funds. Harvard Business Review, 43(1), 63-75.

[65] Vallejo-Jiménez, B, and F. Venegas-Martínez (2017). Optimal Consumption and Portfolio Rules when the Asset Price is Driven by a Time-Inhomogeneous Markov Modulated Fractional Brownian Motion with Multiple Poisson Jumps. Economics Bulletin, 37(1), 314-326.

[66] Vallejo-Jiménez, B., F. Venegas-Martínez, and Y. V. Soriano-Morales (2015). Optimal Consumption and Portfolio Decisions when the Risky Asset is Driven by a Time-Inhomogeneous Markov Modulated Diffusion Process. International Journal of Pure and Applied Mathematics. 104(2), 53-362.

[67] Venegas-Martínez, F. y B. González-Aréchiga (2000). Mercados financieros incompletos y su impacto en los programas de estabilización de precios: el caso mexicano. Momento Económico, 111, 20-27.

[68] Venegas-Martínez, F., G. A. Agudelo-Torres, L. C. Franco-Arbeláez, y L. E. Franco-Ceballos (2016). Precio del dólar estadounidense en el mundo. Procesos de Itô económicamente ponderados en un análisis espacial. Economía y Sociedad, 20(34), 83-105

[69] Venegas-Martínez, F y A. Rodríguez-Nava (2010). Optimal Portfolio and Consumption Decisions under Exchange Rate and Interest Rate Risks: A Jump-Diffusion Approach. Contaduría y Administración, 230, 9-24. 
[70] Venegas-Martínez, F. (2001). Opciones, cobertura y procesos de difusión con saltos: Una aplicación a los títulos de GCARSO. Estudios Económicos, 16(32), 203-226.

[71] Venegas-Martínez, F. (2008). Riesgos financieros y económicos: Productos derivados y decisiones económicas bajo incertidumbre, 2da. Edición. Cengage Learning, México.

[72] Venegas-Martínez, F. (2000). On Consumption, Investment, and Risk. Economía Mexicana, Nueva Época, 9(2), 227-244.

[73] Venegas-Martínez, F. (2001). Temporary Stabilization: A Stochastic Analysis. Journal of Economic Dynamics and Control, 25(9), 1429-1449.

[74] Venegas-Martínez, F. (2006). Stochastic Temporary Stabilization: Undiversifiable Devaluation and Income Risks. Economic Modelling, 23(1), 157-173.

[75] Venegas-Martínez, F. (2009). Temporary Stabilization in Developing Countries and Real Options on Consumption. International Journal of Economic Research, 6(2), 237-257.

[76] Wu, H., and Z. Li (2011). Multi-Period Mean-Variance Portfolio Selection with Markov Regime Switching and Uncertain Time-Horizon. Journal of Systems Science and Complexity, 24(1), 140-155.

[77] Yu, J. (2014). Optimal Asset-Liability Management for an Insurer under Markov Regime Switching Jump-Diffusion Market. Asia-Pacific Financial Markets, 21(4), 317-330

[78] Zariphopoulou T. (2001). A Solution Approach to Valuation with Unhedgeable Risks. Finance and Stochastics, 5(1), 61-82.

[79] Zariphopoulou, T. (1999). Optimal Investment and Consumption Models with Non-Linear Stock Dynamics. Mathematical Methods of Operations Research, 50(2), 271-296.

[80] Zariphopoulou, T. (1992). Investment-Consumption Models with Transaction Fees and Markov-Chain Parameters. SIAM Journal on Control and Optimization, 30(3), 613-636.

[81] Zhang, Q. and G. Yin (2004). Nearly-Optimal Asset Allocation in Hybrid Stock Investment Models. Journal of Optimization Theory and Applications, 121(2), 419-444.

[82] Zhou, X. Y., and G. Yin (2014). Markowitz's Mean-Variance Portfolio Selection with Regime Switching: A Continuous-Time Model, SIAM Journal on Control and Optimization, 42(4), pp. 1466-1482. 